\title{
What is science?
}

Helen Quinn is a theoretical particle physicist at SLAC. Throughout her career, she has been passionately involved in science education and public understanding of science.

In talking about science, whether to the public or to students, we scientists often assume that they share with us a common idea of science. In my experience that is often not the case. To oversimplify, scientists think of science both as a process for discovering properties of nature, and as the resulting body of knowledge, whereas most people seem to think of science, or perhaps scientists, as an authority that provides some information-just one more story among the many that they use to help make sense of their world.

Can we close that gap in understanding? Middle school teachers typically spend a day or so teaching something called the scientific method, but the process by which scientific ideas are developed and tested is messier and much more interesting than that typical capsule description. Some remarkable features of the process are seldom stressed in teaching science, nor are they addressed in explaining any one piece of science to the public. My goal in this column is to provide some ideas for closing that gap in understanding, and to encourage scientists and teachers to communicate about the process as they discuss scientific work.

\section{Eradicating inconsistencies.}

Readers of Physics Today know that science is a process, based on interpretation of experimental or observational data using models and theories, within a tightly constrained logical structure. The constraints arise from needing a logically self-consistent explanation of multiple 
phenomena. Any apparent contradiction between different theories or models, between evidence and theory, or between different sources of evidence must be examined and resolved. Asking questions is a big part of doing science, and choosing to pursue answers to the more compelling and productive ones helps shape a given field. Eventually something resembling an answer might emerge, only to be tested against further observations, models, or theories, with this process often leading to further questions. The work continues, iteratively refining both the theory or model and the questions being examined. Iterations are essential because of the inherent messiness of the process. There are many false starts, with misinterpretations and incomplete information sets sometimes sending science off on a wild goose chase for a while. We scientists could well be more forthright about the fits and starts of research; after all, clearing up the inconsistencies is what conveys much of the authority on the results.

Much of science seeks to explain observations of the current state of the natural world by developing an evidence-based history of how that situation arose, much as a detective reconstructs a crime. Computer programs that can simulate the progression of the system (or some aspects of it) over time are important tools in such science and can be powerful means to predict outcomes. The developed history must be consistent not only with all that is known about the system in question but also with all that is understood about processes that occur within the system. Geoscience, climate science, astrophysics, cosmology and evolutionary biology all use this important history-building approach to develop major parts of their theories.

Theories and models develop over time. Based on data, they undergo a long-term process of testing and refinement before becoming accepted scientific explanations or tools within a given domain. Contrast this with the usual description of the "scientific method," which reduces continuous 
and iterative theory building to the idea that one makes and tests hypotheses. The use of a broad theoretical framework within which each hypothesis must fit, and that gets refined by each test, is generally lacking in the text-book account.

Scientific theories, even when generally accepted after much testing and refinement, are still never complete. Each can be safely applied in some limited domain, some range of situations or conditions for which it has been well tested. Each might also apply in some extended regime where it has yet to be tested, and has little or nothing to offer in still more distant domains. That is the sense in which no theory can be proven to be true; truth is too complete a notion. We need to emphasize that the incompleteness of theory in no way compromises the stability over time of well-established understanding in science-an important notion that is seldom made explicit.

\section{How science progresses}

As we all know, science's a cumulative and expanding knowledge base about the natural world is built not only on the exploration of phenomena in natural or laboratory situations but also on the development of theories and through repeated cycles of carefully designed experiments and observations that test and expand those theoretical ideas. At the core of science lies well-tested theory, at its edges speculation and questions. Authority for a scientific theory comes from explaining lots of data coherently within its tested domain, beyond which it functions as a hypothesis until tested.

Scientists are always working to extend their theories, to understand new domains, to make new connections. New tools extend the domains that can be studied. New discoveries and their explanations almost always 
generate new questions. It may seem that science is ever changing, and indeed at the forefront it is, but over time the storehouse of well-tested and stable scientific knowledge grows.

One key to progress in science is an eye for contradictions and an insistence that they must be resolved. This can make scientists seem overly dogmatic or argumentative in the eyes of a non-scientist because it diverges strongly from usual human behavior. Of necessity, we all live lives full of contradictory elements and we must act without sufficient knowledge to draw logical conclusions, or else become incapable of action. For example, when driving my car I am alert for other drivers changing lanes but assume that cars generally travel within their lanes. Without assumptions about the behavior of other drivers I could not drive in traffic, and yet I know these assumptions are not always true.

Science is done in an artificial environment, where its logic can develop without a need for immediate action. That unnatural environment allows science to yield powerful and unexpected new options for eventual action. It is important to note, however, that some applications of science, such as medicine, cannot wait until all questions are resolved. While medical practice can be based on the best available scientific knowledge and theory, it must often apply them in untested regimes. Much of the public's feeling that science is always changing its conclusions comes from changes in medical advice that occur when new scientific knowledge overrides the previously best guesses of medical practice.

\section{The limits of science}

In everyday usage the question "Why?” can ask either about the mechanism by which something occurred or about the reasons for or 
purposes behind an action. Thus the distinction between reason and mechanism, or between effect and purpose, is often blurred. Religion and philosophy are interested in reasons and purposes, but science cares only about mechanisms. That apparent reduction of the goal is a powerful step that separates modern science from its ancestor, natural philosophy. Modern science focuses our attention on just those questions that can have definitive answers based on observations. Where science does find a path to compare theory with observations, the theories so developed provide a powerful way to understand the world and even to make some predictions about the future. Science provides us with new options that may be applied-for example in technology and medicine-to change the way we live and extend our capabilities. However, scientists tend to forget that issues of reason and purpose are central to many people's questioning, so the answers they get from science seem inadequate.

Applying established scientific theory to new realms can take us beyond the realm of testable science. For an idea to be outside of the domain of science requires not just that no experiment or observation that could test it is feasible with current technology, but that there is no phenomenon that could ever be observed that could illuminate the subject. Physicists' speculations about universes outside our own observable universe (speculations that I find both fascinating and plausible) are extensions into a realm where tests are impossible, because those other universes are, by definition, outside of anything we might ever observe. Such implications from scientific knowledge are not quite at the same level as scientific knowledge in testable domains. I call such speculation scientific metaphysics, distinguishing it from much metaphysical speculation that is clearly not scientific and even contradicts known science. The speculations of scientific metaphysics are constrained and illuminated by the logic of science; they emerge from the same theories that bear on our observable universe and its history. They can lead to new ideas that inform 
theories of observable phenomena. Some physicists may see the term metaphysics as pejorative, but I do not intend it in that way; I merely wish to distinguish this thinking from that which can be constrained by observations.

Going yet further beyond science, moral and ethical questions are not amenable to the methods of science because they do not ask about the natural world. That does not mean that scientists can disregard questions about value, nor that science is irrelevant to making moral and ethical decisions. For example, science can help predict the outcomes of various actions, and thereby give us a longer or deeper view of the consequences of our choices. Science and scientists must be guided by and contribute to the moral and ethical judgments of society in making decisions about which questions to pursue and whether or how to limit that pursuit. Then too, science can present new moral quandaries by offering choices never before available.

\section{A few further thoughts}

Science has its own internal values. Science requires absolute honesty about acquired data, and the intellectual honesty that insists on resolving logical contradictions. Scientists must be open to new ideas and ready to modify their opinions if and when contradictory evidence emerges. Those key values of honesty and openness are essential for science to progress. Scientists are human: they jump to conclusions, they make mistakes in recording or analyzing data. Sometimes a scientist fakes data and commits scientific fraud. The principle of verification by independent replication of experimental results is an important part of science, because it can unmask such errors or fraud. 
Science has tremendous patience for detail. By examining the details we learn the inadequacies of even our most successful theories and how we must change them. Scientists eagerly seek the details that have not been tested and hope for a conflict with their best available theory, because that provides an opportunity for new understanding to emerge. Skeptical testing and retesting of ideas is central to the way science works. Only when every possible detail has survived multiple tests can we say that a theory is well-established, and then only in that regime where the tests were passed.

Their passion for detail sometimes makes scientists' communication difficult for lay people and students. Very often, scientists present so much detailed information that even the most interested listener gets lost in it. Although very important to the progress of science, such details can overwhelm or bore the non-expert. In everyday life, insistence on the way every detail is to be understood is viewed as overly pedantic and generally irrelevant.

Key features of the process of science-questioning, theory building, resolving contradictions, and seeking data to test ideas-are common to all natural science. What is learned in one area often has application in another. That commonality is often hidden as students learn science. Disciplinary curricula too often stress the particulars of each sub-domain more than the underlying methodology or the interconnections. But scientific ideas are not independent of fragments of knowledge; a critical test for any new idea is to examine how it fits together with what is already known. Over time, the network of theories developed for separate domains has become deeply interconnected. In much of today's research, physics cannot be separated from chemistry or biology or Earth science. The separate threads weave together to form a tapestry, all the richer for the multiplicity of its details and approaches. The process is certainly 
messy but unquestionably powerful. Scientists and science teachers need to do a better of job of communicating both aspects. 